\title{
Polineuropatía tardía inducida por órganos fosforados. Reporte de un caso y revisión de la literatura
}

\section{Induced late polineuropathy by products containing phosphate. A case report}

\author{
PAMO Oscar ${ }^{1}$, BANDRES Pilar ${ }^{2}$, LOJA David $^{3}$ \\ ${ }^{1}$ Internista del Hospital Loayza de Lima y profesor de la Universidad Peruana Cayetano \\ Heredia. \\ ${ }^{2}$ Internista del Hospital Loayza de Lima \\ ${ }^{3}$ Residente de Medicina Interna, Hospital Loayza de Lima-UPCH.
}

En nuestro medio no son infrecuentes las intoxicaciones por órganos fosforados (OF) y, por lo general, no constituyen un problema diagnóstico ni de tratamiento. La casi totalidad de estos casos son intoxicaciones agudas debido a la ingestión con fines suicidas, a la exposición durante fumigaciones $\mathrm{y}$ ocasionalmente, a la ingestión accidental.

El compromiso neurológico en las intoxicaciones agudas por órganos fosforados es conocido (1-5). En cambio, los reportes de compromiso neurológico tardío son infrecuentes. En nuestro medio se reportó una polineuropatía con carácter epidémico y se asumió que la causa fuera una intoxicación por OF's usados en fumigaciones sin contar con pruebas concluyentes (6).

A continuación se presenta una paciente que desarrolló una polineuropatía tardía y los estudios evidenciaron una exposición a un OF.

\section{CASO CLINICO}

H.C. " 602462.J.C.Ch., mujer de 28 años, conviviente, cocinera/lavandera, natural y procedente de Piura, ingresó al Hospital Loayza de Lima el 25 de junio de 1993. Mal informante.

Refería un tiempo de enfermedad de cinco semanas. Estando con cinco meses de gestación, a mediados de mayo presentó náuseas, vómitos y fiebre. Acudió al Hospital de Piura donde se le hizo los diagnósticos de Encefalopatía de Wernicke, óbito fetal y sepsis. Se realizó la extracción del óbito fetal y legrado uterino. La paciente recuerda que por aquel entonces comenzó a notar la disminución de la fuerza en los miembros, especialmente en los inferiores, incontinencia urinaria y fecal, mareo y disartria. La paciente fue dada de alta y la madre la trajo a Lima para el tratamiento de las molestias aparecidas.

En su ingreso al hospital la paciente refería que la debilidad muscular era consecuencia de la anestesia a que fue sometida para la intervención quirúrgica.

Funciones biológica: apetito y sed conservados; orina y heces: no controla esfínteres; sueño: conservado. 
Antecedentes fisiológicos: de crecimiento y desarrollo normales. Antecedentes ginecológicos: menarquia a los 12 años; grávida 2, para 1. Antecedentes patológicos: convulsiones tónico-clónicas a los 9 años de edad, tomó fenitoína irregularmente años atrás.

Antecedentes familiares y epidemiológicos no contributorios.

En el examen físico: P.A.: 90/60, pulso 80/min., F.R. 20/min. T $37^{\circ}$. Paciente en regular estado general, hidratada, lúcida, en decúbito dorsal pasivo.

Piel sin lesiones dérmicas características. Marcada disminución del tejido celular subcutáneo. Huesos y articulaciones de caracteres normales. Masas musculares disminuidas. No adenomegalias. Cráneo normocéfalo. Oídos, ojos, nariz, boca y faringe de caracteres normales. Cuello sin alteraciones. Pulmones murmullo vesicular normal. Mamas péndulas, sin alteraciones. Corazón con ruidos cardíacos normales. Abdomen blando y si vísceromegalia. Tacto rectal con esfínter normotónico, ampolla rectal con contenido fecal. Sistema nervioso: lúcida, bradilálica. Fondo de ojo normal y no se evidenció compromiso de los pares craneales.

Cuadriparesia, a predominio distal, mas acentuado en miembros inferiores y asimétrico. Arreflexia generalizada y sin compromiso de la sensibilidad táctil dolorosa.

Exámenes auxiliares: Hb 12.9; Hemograma: leucocitos 11400 pmmc con leve neutrofilia; glicemia $81 \mathrm{mg} / 100 \mathrm{ml}$ : creatinina $0.74 \mathrm{mg} / 100 \mathrm{ml}$; Alb $3.51 \mathrm{~g} / 100 \mathrm{ml}$, Glob $2.72 \mathrm{~g} / 100 \mathrm{ml}$; crioglobulinas neg.; Na $140 \mathrm{mEq} / \mathrm{L}, \mathrm{K} 3.34 \mathrm{mEq} / \mathrm{L}, \mathrm{Cl} 103$ $\mathrm{mEq} / \mathrm{L}, \mathrm{HCO} 27.7 \mathrm{mEq} / \mathrm{L}$; $\mathrm{pO}_{2} 75 \mathrm{~mm} \mathrm{Hg}, \mathrm{pCO}_{2} 43 \mathrm{mmHg}, \mathrm{pH}$ 7.42, Sat. O2 95.2 mmHg. Examen de orina: $\mathrm{D}=1,020, \mathrm{pH}$ ácido; leucocitos 60-80 /campo, 10\% aglutinados y degenerados, hematíes 2-3/campo; cultivo: más de 1'500,00o col $/ \mathrm{ml} \mathrm{de}$ Klebsiella sp; L.C.R.: células $10 \mathrm{LMN} / \mathrm{mmc}$, proteínas $23 \mathrm{mg} / \mathrm{ml}$, glucosa $51 \mathrm{mg} / \mathrm{ml}$, Gram, BAAR y criptococucs negativos, cultivo negativo.

Test de Watson Schwartz negativo. Proteína de Bence Jones en orina negativo. HIV (ELISA-Abbott) negativo.

Velocidad de conducción y electromiografía: 1- no se registró latencia distal motor en el nervio peroneal común derecho, fueron normales en el tibial posterior, mediano y cubital derechos.

Asimismo sus velocidades de conducción motora fueron normales. 2- Ausencia de latencia distal sensitiva en el nervio sural derecho. Fueron normales en los nervios cubitales y medianos derechos. 3- Ausencia de potenciales de acción muscular voluntarios con abundantes fibrilaciones y ondas positivas en el tibial anterior, pedio y vasto interno derechos; y disminución de los potenciales con signos de denervación en gemelo, recto anterior, abductor corto pulgar, primer interóseo dorsal derechos.

En conclusión: polineuropatía mixta, sensitivo-motora, de tipo axonal y de grado severo que compromete los cuatro miembros a predominio distal.

Análisis toxicológico en sangre (realizado en el Centro de Información hay Control Toxicológico, Facultad de Farmacia y Bioquímica-UNMSM, Av. Grau \# 800 CICOTOX): positivo para insecticidas órganos fosforados $y$ negativo para 
organoclorados, organocarbámicos, barbitúricos, fenotiazínicos, benzodiazepinas, alcaloides (cocaína), cumarinas, alcohol, arsénico, hexaclorobenceno, plomo y talio.

Cuantificación de órgano-fosforados: $4694 \mathrm{mg}$ de dicarzol $/ \mathrm{ml}$; actividad de colinesterasa eritrocítica: $0.61 \mathrm{Y} / \mathrm{h}$ (V.N.: $0.68-1.04 \mathrm{U} / \mathrm{h}$ ); actividad de esterasa nerotóxica: $171 \mathrm{UI} / \mathrm{ml}$ (V.N.: $221 \mathrm{UI} / \mathrm{ml}$ en mujeres).

Durante su permanencia en el hospital la paciente mostró una conducta pueril que correspondía a su personalidad previa antes que a secuelas de la intoxicación. Básicamente se le brindó terapia física. Seis meses después, la paciente presentaba una leve recuperación de la función motora, logrando sostenerse de pie con ayuda. La paciente negó en todo momento la ingestión voluntaria de OF's pero no se descartó la posibilidad de que lo haya hecho de manera subrepticia.

\section{DISCUSIÓN}

La intoxicación por OF's es, probablemente, la más estudiada desde que se usaron como insecticidas o plaguicidas en los campos de cultivos y se reportaron las diversas manifestaciones muscarínicas y nicotínicas en los humanos expuestos.

Las manifestaciones clínicas derivadas de la intoxicación aguda por OF"s y que corresponden al sistema nervioso por lo general quedan enmascaradas por las manifestaciones de los otros aparatos y sistemas que suelen ser más llamativas y espectaculares.

Los primeros síntomas son de tipo muscarínicos (miosis, sialorrea, hipersecreción bronquial, bradicardia, visión borrosa, etc). Aproximadamente unas 4 horas luego de iniciada la intoxicación aparecen los síntomas nicotínicos, sobre todo las fasciculaciones y parálisis músculoesqueléticas debidas al bloqueo en la placa mioneural que origina una debilidad severa que amenaza la vida. En este sentido, antes que la determinación de la actividad de la colinesterasa, los estudios electrofisiológicos tienen una alta sensibilidad en el diagnóstico, en la recuperación, en la predicción de los pacientes que deben recibir y ser retirados de la ventilación mecánica. Así, los potenciales de acción muscular compuestos evocados únicos espontáneos y repetidos son los indicadores más tempranos y sensibles de la inhibición de la acetilcolinesterasa; un decremento de estos potenciales evocados luego de la estimulación nerviosa repetida es la alteración más severa. En lo máximo de la intoxicación ningún potencial fue evocado luego de los primeros estímulos. El fenómeno decremento-incremento sólo ocurrió en los estadíos leves de intoxicación y son característicos de la inhibición de la acetil-colinesterasa (7).

La colinesterasa es la enzima que hidroliza a la acetilcolina y existen dos tipos: la acetilco-linesterasa que se encuentra en el sistema nervioso y en los eritrocitos; y, la plasmática o pseudocolinesterasa que se encuentra también en el tejido nervioso y en el plasma. Como ambas enzimas son inhibidos por lo OF's además que la actividad de la pseudocolinesterasa varía en muchas situaciones, una disminución de la actividad de la colinesterasa eritrocítica es más específica para indicar una exposición a los OF's. La actividad de la colinesterasa eritrocítica se recupera en un $1 \%$ diario mientras que la plasmática se recupera en un $25 \%$ en los primeros 7 a 10 días, siempre que no ocurra reexposición. Una disminución del $25 \%$ o más de la actividad de la colinesterasa eritrocítica puede permanecer disminuida hasta 90 y 120 días antes de retornar a cifras normales (8). 
Entre las manifestaciones neurológicas persistentes luego de una intoxicación aguda han sido descritos cambios EEG's persistentes por varias semanas; irritabilidad, nerviosismo, fatiga, letargo, dificultad para memorizar; confusión, falta de concentración, mialgias, calambres, parestesias, y debilidad de miembros; reacciones esquizofrénicas, depresivas o maníacas (9); bajo rendimiento en los tests de evaluación verbal y visual, memoria visual, velocidad visomotora, seguimiento y solución de problemas, uniformidad motora y destreza (10).

Se ha demostrado que la exposición repetida a los OF's origina a una disminución de los receptores colinérgicos muscarínicos en el cerebro y en los tejidos periféricos y se piensa que estos cambios tienen que ver con el desarrollo de la tolerancia a los OF's y que pueden tener un papel en las disfunciones cognitivas luego de la exposición repetida a los OF's.

También se han descrito cuadros neurológicos tardíos, especialmente de tipo polineuropático. A esta entidad se le ha denominado la polineuropatía tardía inducida por órganos fosforados-PTIOF (organophosphate induced delayed polyneuropathyOPIDP). Mientras que las manifestaciones de las crisis colilnérgicas son debidas a inhibición de la esterasa neurotóxica (ENT). Inicialmente se produce a la fosforilación de la ENT en el tejido nervioso.

Luego sucede una rápida reacción que incluye la pérdida de un grupo ligado al fósforo dejando un grupo ligado a la enzima y cargado negativamente.

A este proceso se le conoce como "envejecimiento" de la enzima. Los OF's que producen este "envejecimiento" causaran la polineuropatía tardía. Experimentalmente, un alto umbral de inhibición de la ENT, 70 a 80\%, del tejido nervioso producirá la PTIOF dos semanas más tarde. El umbral de inhibición de ENT en los humanos se desconoce. No se conoce la actividad fisiológica de la ENT pero su determinación en los linfocitos correlaciona con la medida en el tejido nervioso, de aquí que puede ser empleado como prueba diagnóstica y pronostica en el caso de intoxicaciones agudas con OF's conocidos de producir la PTIOF $(11,12)$.

Esta polineuropatía tardía suele presentarse una a tres semanas después de la exposición aguda o en un tiempo variable cuando ocurre exposición crónica o prolongada (11). El cuadro clínico es el de una polineuropatía sensitivo-motora, pero predominantemente motora, que también puede comprometer el sistema nervioso central, si alteraciones en el LCR ycon estudios electrofisiológicos que demuestran degeneración axonal primaria y de la mielina secundariamente (12-14).

Entre la intoxicación aguda del sistema nervioso y 1 PTIOF se ha descrito un síndrome intermedio de efectos neurotóxicos. Así, parálisis de los músculos proximales de los miembros, de los flexores del cuello, de los pares craneales y de los músculos respiratorios, dos a tres días después de una reconocida exposición aguda, que puede durar hasta 18 días y recuperarse o evolucionar a la forma tardía, y que parece corresponder a una lesión postsináptica en la placa mioneural (15). Algunos autores han cuestionado este síndrome intermedio. 
La PTIOF tiene una morbilidad importante. Las secuelas neurológicas no siempre son reversibles y dependen del agente tóxico, de la cantidad, del número de exposiciones, etc.

La polineuropatía tardía entre en el diagnóstico diferencial de las otras polineuropatías, especialmente con el síndrome de Guillain-Barre, toda vez que no se cuente con el antecedente de ingestión o exposición a los OF's como fue el caso de nuestra paciente y que nos obligó a una búsqueda

minuciosa para determinar la causa. No creemos que la paciente tuvo el síndrome de Wernicke sino que fueron las manifestaciones iniciales de la polineuropatía tardía.

El tratamiento de la PTIOF consiste básicamente en fisioterapia y en medidas de sostén que pueden llegar a la ventilación mecánica. La hemoperfusión, a través de carbón activado, no ha demostrado ser eficaz en la remoción de los OF's debido a la alta solubilidad de estos agentes en el tejido adiposo y por la fuerte ligazón al compuesto de las acetilcolinesterasas (16).

Con el presente caso queremos resaltar que este cuadro clínico bien definido e infrecuente como causa de polineuropatía pero que puede ser determinado por los estudios respectivos.

\section{REFERENCIAS BIBLIOGRÁFICAS}

1.Wadia RS, Sadagopan C, Amin RB y Sardesai HV. Neurological manifestations of organophosphorous insecticide poisoning Neurosurg psychiat 1974; 841-7.

2.Senayake N, y Jeyaratnam J. Toxic polyneuropathy due to gingili oil contaminated with thr-cresyl phosphate affecting adolescent girls Sri Lanka. Lancet Jan 10, 1981; 889.

3.Yelamos F, Diez F, Martín C. et al. Med Clin (Barc) 1992; 98: 681-4.

4.Fisher JR. Guillain Barre Sindrome following organophosphate poisoning. JAMA 1977; 238(1*): 1950-1.

5.Senanayake N y Johnson MK. Acute polyneuropathy after poisoning by a new organophosphate insecticide.N Eng J Med 1882; 306(3): 155-7.

6.Voto Bernales J, Fernández, E, Mejía C, et al. Polineuritis toxoepidemica en Paramonga. Revista Médica del Hospital Obrero 1957; 21-94.

7.Besser, Gutmann L, Dillman U et al. End-plate dysfunction in acute organophosphate intoxication. Neurology 1989; 39: 561-7.

8.Milby TH. Prevention and management of organophosphate poisoning. JAMA 1971; 216(13): 2131-3.

9.Namba $\mathrm{T}$, Nolte Ct, Jackreld $\mathrm{J}$ y Grob D. Poisoning due to organophosphate insecticide, Am J Med 1971; 50: 475-92.

10.Rosenstock L, Keifer M, Daniel WE et al. Chronic central nervous system effects of acute organophosphate pesticide intoxication. The pesticide Health Effects Group. Lancet 1991; 338(8761): 223-7.

11.Lotti M, Becker Ch E y Aminoff MJ. Organophosphate polyneuropathy: athogenesis and prevention. Neurology 1984; 34: 658-62

12.Abou-Donia MB. Organophosphorus ester-induced delayed neurotoxicity. Ann Pharmacol Toxicol 1981; 21: 511-48.

13. Gadoth N, y Fisher A. Late onset of neuromuscular block in organophosphorous poisoning. Ann Int Med 1978; 88(5): 654-5. 
14. Vasilescu C, Alexianu M y Dan A. Delayed neurophaty after organophosphorus insecticide (Dipterex) poisoning: a clinical, electrophisioloical and nerve biopsy study. J Neurol Neurosurg Psych 1984; 47: 543-8.

15. Senayake N y Karalliedde L. Nerotoxic effects of orgaophosphorus insecticides. An intermediate syndrome. N Eng J Med 1987; 316(13): 761-3.

16. Martínez Chuecos J, Jurado MC, Paz M et al. Experience with hemoperfusión for organophosphate poisoning. Critical Care Medicine 1992; 20(11): 1538-43. 